\title{
An Analysis of Read-Out Perturbations Seen on an Analytical Balance with a Swinging Pan
}

\author{
Marvin E. Cage* and Richard S. Davis*
}

National Bureau of Standards, Washington, DC 20234

September 8,1981

\begin{abstract}
An analysis of the dynamic behavior of a single-pan mechanical balance is presented. In particular, errors caused by a swinging pan are analyzed in detail. Results point to a large effect which, though apparently not previously appreciated, is nevertheless easily verified experimentally. It is suggested that this effect can be reduced to insignificance in a balance whose beam is servo-controlled to an angle perpendicular to the local gravitational field.
\end{abstract}

Key words: Analytical balance; balance dynamics: balance sensitivity; balance suspension; knife-edge bearings; Mathieu's equation; single-pan balance.

\section{Introduction}

The single-pan analytical balance has become standard equipment in laboratories performing precise mass determinations. The mechanical balances with which this paper is concerned include those having built-in dial weights, a damped beam, and an optical scale which is directly related to beam angle and which has a range equal to or greater than the increment of the smallest dial. Many commercial models of this type of balance achieve a precision of better than $0.5 \times 10^{-6}$ of maximum load. Indeed, the most precise kilogram comparator-an undamped version of these balances-achieves a relative precision of $1 \times 10^{-9}[1] .^{1}$ Equations derived below also treat the case of undamped balances.

It is a common experience of users of one-pan analytical balances that, occasionally, a badly-centered load will cause the pan to swing which, in turn, produces an oscillation of the optical-scale indication. When this occurs, one generally discards the reading and repeats the operation with more care. The question we posed, however, was: By what physical process does the pan-swing manifest itself as an oscillation of the balance beam? The answer might lead to the minimization of the unwanted effect.

The only previous attempt, to our knowledge, at addressing this question has been in a paper by Bowman and Macurdy [2]. These authors reject attempting an analytical solution to the dynamic errors associated with a swinging pan because such a solution would be difficult except under unrealistic conditions. Instead, they point out that a significant part of the dynamic swing-error is due to the centripetal acceleration of the pan. The maximum amplitude of this acceleration is $g \gamma^{2}$ for small $\bar{\gamma}$, where $g$ is the local acceleration of gravity and $\gamma$ is the peak angular amplitude of the pan swing [2]. Even qualitatively, however, this component may be excluded as dominant, at least on many analytical balances. The reason is that a swinging pan is seen to produce an oscillation in the optical indication at the same frequency as the pan oscillation, whereas the centripetal acceleration is modulated at twice the frequency of the pan (i.e., the centripetal acceleration passes through two maxima during one complete period of pan swing).

Our approach to obtaining a solution to the question posed above has been to generate a complete analytical solution to the equations of motion of an idealized single-pan balance. Our idealized balance operates with knives and flats acting as pivot bearings. The knives do not have perfectly sharp edges but instead have finite radii of curvature. This complexity was added to help elucidate the role of imperfect bearings in the dynamic behavior of the balance.

While our balance model, as will be seen below, requires definite restrictions (such as small-angle motions) and contains some unrealistic assumptions (such as perfectly parallel knives), it nonetheless includes many

- Center for Absolute Physical Quantities, National Messurement Laboratory.

' Figures in brackets indicate literature references at the end of this paper. 
of the fundamental physical aspects of the problem and is amenable to analysis. Semiquantitative predictions of the dynamic behavior of analytical balances can be made based on solutions of the model. These predictions have been verified experimentally as described in section 5 below.

\section{Equations of Motion}

\subsection{Method}

The equations of motion for a damped, single-pan balance will be obtained by using the Principle of Virtual Work and D'Alembert's Principle [3, 4]. The suspension system, the balance beam, and the counterweight are initially represented by point masses located at their respective centers of gravity; they will later be treated as distributed masses.

The first step is to determine the coordinates of these point masses as a function of time. This information is used in the Principle of Virtual Work to obtain the generalized torques-and thus the balance beam stiffness and sensitivity expressions. The kinetic energy of the system is also obtained from the equations describing the locations of the point masses. The kinetic energy, the generalized torque, and a velocity-dependent Rayleigh dissipation function [3] are then used in accordance with D'Alembert's Principle to generate the equations of motion for the system.

\subsection{Coordinate System}

Figure I shows the coordinate system for the balance. Note that the $\mathrm{z}$ axis, which is defined to be parallel to the local gravitational field, arbitrarily points downward. The $y$ axis is thus parallel to the direction that the plane, defined by the two knife edge contact points $(P)$ and $(C)$, would have if this plane were at gravitational

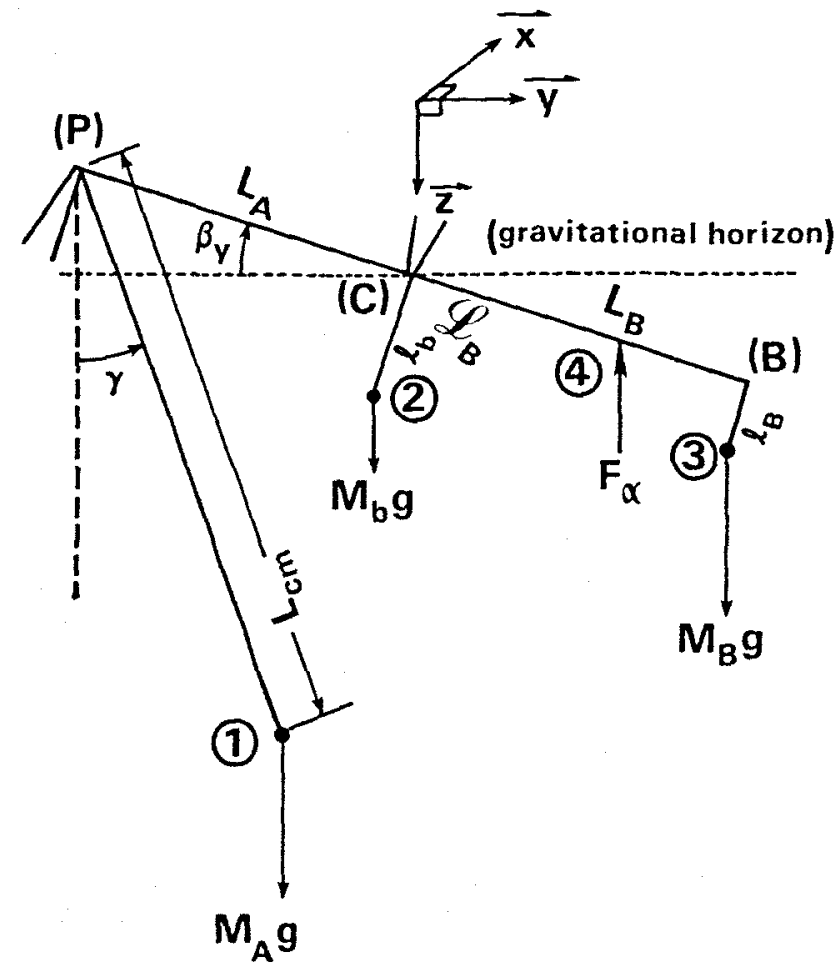

Frcune 1. The coordinate system and quantities used in the equations of motion. The balance beam rotates in the $y-z$ plane, while the suspension system-load mass combination, represented by a simple pendulum, swings either in the $x-z$ or the $y-z$ plane via crossed knives at pivot point $(\mathrm{P})$. The origin is fixed at point (C), which is the center knife contact point for $\beta_{y}=0$. 
horizon; i.e., if $\beta_{y}=0$. The $x$ axis is directed into the figure so that the coordinate system will be righthanded, and the origin of the balance coordinate system is fixed at point $(C)$, which is the center knife contact point for $\beta_{y}=0$. Therefore, the balance beam swings in the $y-z$ plane about gravitational horizon. The time development of $\beta_{y}(t)$ describes this motion.

Points 1, 2, and 3 of figure 1 represent the centers of mass of the suspension system-load mass combination $\left(M_{A}\right)$, the balance beam mass $\left(M_{b}\right)$, and the counterweight mass $\left(M_{B}\right)$, respectively. A velocity-dependent damping force, $F_{a}$, is applied at point 4 . The knife shown at point $(P)$ is actually assumed to be two knives crossed in the same plane (see figure 5 of reference [1] or figure 3 of reference [5]). These crossed knives consist of a load knife, $L$, with a symmetry axis in the $x$ direction and an intermediate knife, $l$, pointing in the $y$ direction. Therefore, the suspension system is free to pivot in any direction about point $(P)$ with an instantaneous angle $\gamma$. If the knives do not cross in the same plane, then the center of mass length, $L_{\mathrm{cm}}$, can be replaced by $\left(L_{\mathrm{cm}}\right)_{x}$ and $\left(L_{\mathrm{cm}}\right)_{y^{*}}$. (The purpose of the intermediate knife in balance design is to compensate for non-parallelism of the two main knives [5]. In our model the main knives are assumed parallel, however. Thus, in our model the only effect of the intermediate knife is to allow a second degree of freedom for a swinging pan.)

Normally, the suspension system would include a gimbal-like device to minimize the positional effects of weights on the scale pan [2]. This would involve a double pendulum on a balance beam-making the problem analytically intractable, and also masking the physical effects. (Actually, the gimbal often has enough friction to quickly dampen the suspension system motion to that of a single pendulum, so the results of this paper still apply to many single-pan balances.)

We will assume the motion of the suspension system to be that of a simple pendulum. In order to gain the maximum physical insight, we consider its motion either to be entirely in the $x-z$ plane or entirely in the $y$ $z$ plane.

Sl units will be used in the derivations, with masses in kilograms, lengths in meters, forces in Newtons, torques in Newton-meters, and angles in radians. Note that the quantities $\gamma_{x}, \gamma_{y}, \beta_{y}, \ell_{b}, \ell_{B}$, and $\mathscr{L}_{B}$ have signs associated with them-and that they are arbitrarily given positive signs in the instantaneous configuration at time $t$ shown in figure 1 . Small angle approximations: $\cos \gamma \approx 1, \cos \beta_{y} \approx 1, \sin \gamma \approx \gamma$, and $\sin \beta_{y} \approx \beta_{y}$ will be made at the appropriate places. The $\gamma$ approximations are made because precision balances cannot function with large suspension system amplitudes, while the $\beta_{y}$ approximations are made in order to obtain analytical solutions.

\subsection{Coordinates of the Balance}

\subsection{1 $\gamma_{x}$ and $\beta_{y}$ Motions}

Assume that the center, load and intermediate knife edges are symmetrically honed cylinders such that they have radii of curvature $\rho_{C}, \rho_{L}$ and $\rho_{l}$, respectively. Then, with the aid of figures $2-4$, the coordinates of points 1,2 , and 3 of figure 1 are:

$$
\begin{aligned}
& x_{1}=L_{\mathrm{cm}} \sin \gamma_{x}-\rho_{I} \gamma_{x} \\
& y_{1}=-L_{A} \cos \beta_{y}-\rho_{L} \sin \beta_{y}+\rho_{C} \beta_{y} \\
& z_{1}=-L_{A} \sin \beta_{y}-\rho_{L}\left(1-\cos \beta_{y}\right)-\rho_{C}\left(1-\cos \beta_{y}\right)-\rho_{A}\left(1-\cos \gamma_{x}\right)+L_{\mathrm{cm}} \cos \gamma_{x} \\
& x_{2}=0 \\
& y_{2}=-\ell_{b} \sin \beta_{y}+\rho_{C} \beta_{y} \\
& z_{2}=\ell_{b} \cos \beta_{y}-\rho_{C}\left(1-\cos \beta_{y}\right) \\
& x_{3}=0 \\
& y_{3}=L_{B} \cos \beta_{y}-\ell_{B} \sin \beta_{y}+\rho_{C} \beta_{y} \\
& z_{3}=L_{B} \sin \beta_{y}+\ell_{B} \cos \beta_{y}-\rho_{C}\left(1-\cos \beta_{y}\right),
\end{aligned}
$$

where $\gamma_{x}$ and $\beta_{y}$ are functions of time. 


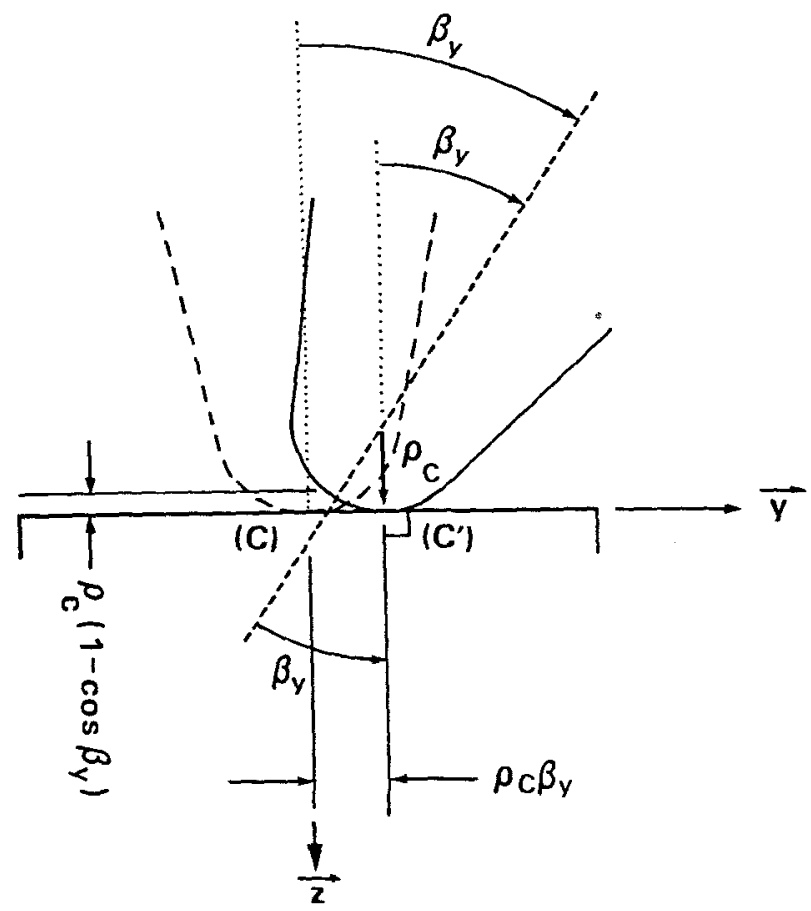

Figure 2. Effect on balance beam of center knife rolling on its flat. The origin is fixed at point $(C)$, with the positive $x$ axis directed into the figure.

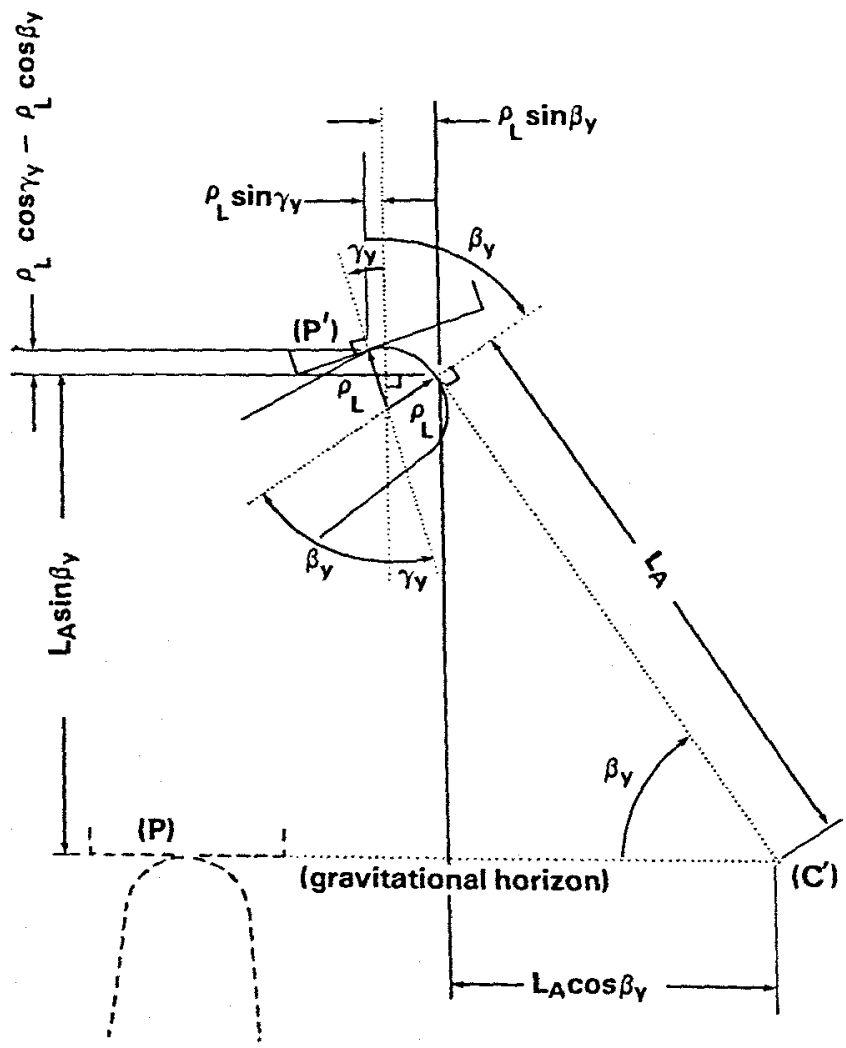

FigLre 3. Effect on balance beam of the load knife flat rolling on the load knife edge. 


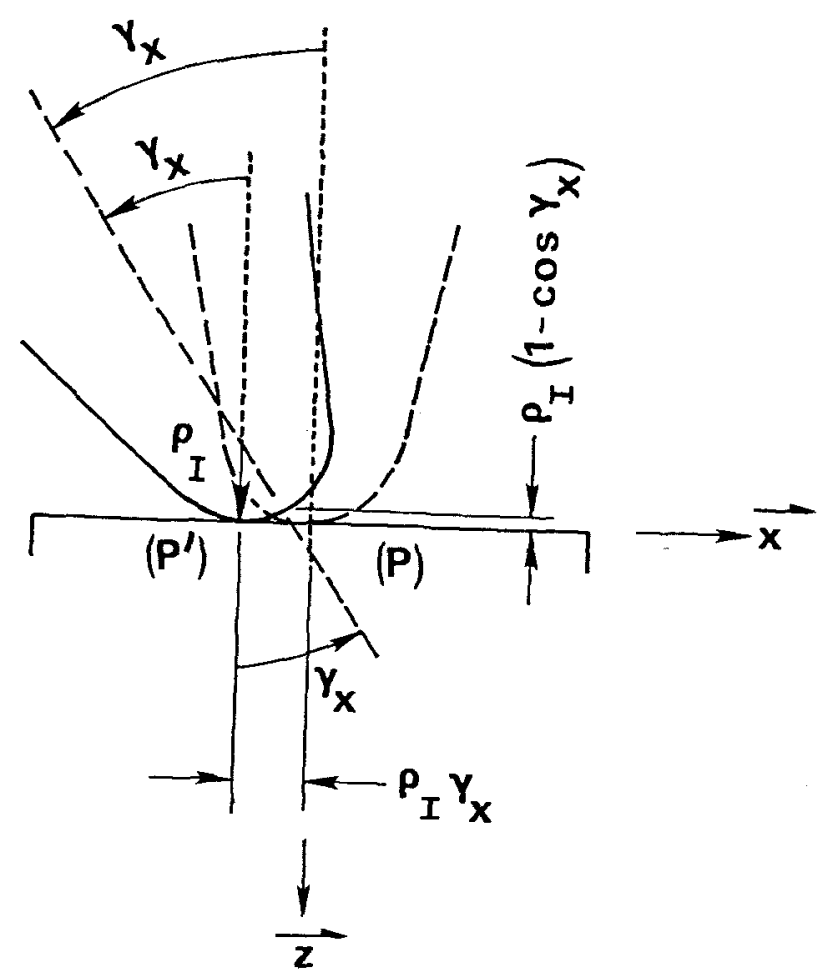

FigurE 4. Effect of the intermediate knife rolling on a flat. The positive $y$ direction, pointing towards the center knife, is out of the figure. For simplicity, the edges of the crossed knives are assumed to lie in a plane.

\subsection{2 $\gamma_{y}$ ond $\beta_{y}$ Motions}

The coordinates of points 1,2 , and 3 of figure 1 are:

$$
\begin{aligned}
& x_{1}=0 \\
& y_{1}=-L_{A} \cos \beta_{y}-\rho_{L} \sin \beta_{y}-\rho_{L} \sin \gamma_{y}+\rho_{C} \beta_{y}+L_{\mathrm{cm}} \sin \gamma_{y} \\
& z_{1}=-L_{A} \sin \beta_{y}-\rho_{L} \cos \gamma_{y}+\rho_{L} \cos \beta_{y}-\rho_{C}\left(1-\cos \beta_{y}\right)+L_{\mathrm{cm}} \cos \gamma_{y} \\
& x_{2}=0 \\
& y_{2}=-\ell_{b} \sin \beta_{y}+\rho_{C} \beta_{y} \\
& z_{2}=\ell_{b} \cos \beta_{y}-\rho_{C}\left(1-\cos \beta_{y}\right) \\
& x_{3}=0 \\
& y_{3}=L_{B} \cos \beta_{y}-\ell_{B} \sin \beta_{y}+\rho_{C} \beta_{y} \\
& z_{3}=L_{B} \sin \beta_{y}+\ell_{B} \cos \beta_{y}-\rho_{C}\left(1-\cos \beta_{y} .\right.
\end{aligned}
$$

\subsection{Principle of Virtual Work}

The Principle of Virtual Work $[3,4]$ states that

$$
\delta W=\sum_{i=1}^{3} F_{i} \cdot \mathbf{d} \ell_{i}=\sum_{i=1}^{3}\left[\left(F_{i}\right)_{x} \delta x_{i}+\left(F_{i}\right)_{y} \delta y_{i}+\left(F_{i}\right)_{x} \delta z_{i}\right]
$$

where $x_{i}=x_{i}\left(\gamma, \beta_{y}\right), y_{i}=y_{i}\left(\gamma, \beta_{y}\right)$ and $z_{i}=z_{i}\left(\gamma, \beta_{y}\right)$, and $i$ represents any of the three points shown in figure 1 . 
Since $\left(F_{i}\right)_{x}=\left(F_{i}\right)_{y}=0$ for this problem, the expression for the virtual work reduces to

$$
\begin{aligned}
\delta W & =\sum_{i=1}^{3}\left(F_{i}\right)_{z} \delta z_{i} \\
& =\sum_{i=1}^{3}\left[\left(F_{i}\right)_{x}\left(\frac{\partial z_{i}}{\partial \gamma_{x}}\right) \delta \gamma_{x}+\left(F_{i}\right)_{z}\left(\frac{\partial z_{i}}{\partial \gamma_{y}}\right) \delta \gamma_{y}+\left(F_{i}\right)_{x}\left(\frac{\partial z_{i}}{\partial \beta_{y}}\right) \delta \beta_{y}\right] \\
& =Q_{\gamma_{x}} \delta \gamma_{x}+Q_{\gamma_{y}} \delta \gamma_{y}+Q_{\beta_{y}} \delta \beta_{y}
\end{aligned}
$$

where $Q_{\gamma_{x}}, Q_{\gamma_{y}}$ and $Q_{\beta}$, are the generalized torques for the $\gamma_{x}, \gamma_{y}$, and $\beta_{y}$ motions. It follows from eq (1) and figure 1 that

$$
Q_{\beta_{y}}=M_{A} g \frac{\partial z_{1}}{\partial \beta_{y}}+M_{b} g \frac{\partial z_{2}}{\partial \beta_{y}}+M_{B} g \frac{\partial z_{3}}{\partial \beta_{y}} .
$$

Analogous expressions are readily obtained for $Q_{\gamma_{x}}$ and $Q_{\gamma_{y}}$, but we will only be concerned with the consequences of torques about the center knife, rather than about the load or intermediate knives, because we are interested in investigating causes of read-out fluctuations of balances.

\subsection{Generalized Torques}

Using the coordinates listed in section 2.3.1 for $\gamma_{x}$ and $\beta_{y}$ motions, and small angle approximations, eq (2) becomes

$$
Q_{\beta}=\left[M_{B} g L_{B}-M_{A} g L_{A}\right]-\left[M_{A} g\left(\rho_{L}+\rho_{C}\right)+M_{b} g\left(\ell_{b}+\rho_{C}\right)+M_{B} g\left(\ell_{B}+\rho_{C}\right)\right] \beta_{y} .
$$

This expression for $Q_{\beta}$, also applies exactly for $\gamma_{y}$ and $\beta_{y}$ motions.

\subsection{Balance Beam Stiffness and Sensitivity}

Assume the balance beam is in static equilibrium; therefore, $Q_{\beta_{v}}=0$, and (3) becomes

$$
\left[M_{B} g L_{B}-M_{A} g L_{A}\right]=\left[M_{A} g\left(\rho_{L}+\rho_{C}\right)+M_{B} g\left(\ell_{b}+\rho_{C}\right)+M_{B} g\left(\ell_{B}+\rho_{C}\right)\right] \hat{\beta}_{y}
$$

which is of the form $\hat{\Gamma}_{\beta,}=\kappa \hat{\beta}_{y}$, where $\hat{\Gamma}_{\beta}$, is the torque about the center knife, the symbol "n" represents equilibrium values, and $k$ is the balance-beam stiffness:

$$
\kappa=\left[M_{A} g\left(\rho_{L}+\rho_{C}\right)+M_{b} g\left(\ell_{b}+\rho_{C}\right)+M_{B} g\left(\ell_{B}+\rho_{C}\right)\right]
$$

This result is the single-pan analogue to the stiffness of a two-pan balance with knives of finite radius [6, 7].

The balance sensitivity, $S$, to changes in torque is

$$
S=\frac{\Delta \hat{\beta}_{y}}{\Delta \hat{\Gamma}_{\beta_{v}}}=\frac{1}{\kappa},
$$

and has the dimensions of $\mathrm{rad} / \mathrm{N} \cdot \mathrm{m} .^{2}$

\footnotetext{
${ }^{2}$ Note that if the balance has ideal bearings $\left(\rho_{L}=\rho_{C}=0\right)$ and if the position of the counter-poise is adjusted so that $\ell_{B}=0$, the sensitivity is conipletely independent of $M_{3}$ and $M_{3}$. This result seems to be in contradiction to eq $(T)$ of $[7]$ and to one statement made in that paper. The formulation of [7] can be reconciled with otr own if one realizes that the coordinate system of [7] is itself a function of $h_{g}$. Hence, for balances whose construction is close to ideal (i.e., $\left.l_{a}<<\left(M_{b} / M_{g}\right) \ell_{b}\right)$, the static properties (such as sensitivity) do not depend strongly on load. The dynamic properties (such as period of the balance) do depend strongly on load: II a $1-\mathrm{kg}$ single-pan mass comparator is converted to a 2-kg comparator by doubling the mass of the counterpoise. the sensitivity of the balance will not change appreciably provided the beam and knives do not deform under this modification. The period of the undamped balance will increase markedly, however. (A clear and succinct discussion of this point can be found in section 3.A of reference [12]).
} 
Note that the balance sensitivity depends upon both $\ell_{b}$ and $\ell_{B}$-and that in general neither their individual magnitudes nor signs are known. This does not matter, however, since the sensitivity is a directly measurable quantity.

Equation (3) can be rewritten as $Q_{\beta_{y}}=\left[M_{B} g L_{B}-M_{A} g L_{A}\right]-\kappa \beta_{y}$. Therefore, the effective changes in lever arms $L_{A}$ and $L_{B}$-due to the knife edges rolling on their flats-enters into the quantity $\kappa \beta_{y} ;$ also, $\kappa$ and $S$ will not be constant if the effective values of $\rho_{C}$ and $\rho_{L}$ vary with $\beta_{y}$.

\subsection{Kinetic Energies}

\subsection{1 $\gamma_{x}$ and $\beta_{r}$ Motions}

The kinetic energy of the system is

$$
\begin{gathered}
T=T_{1}+T_{2}+T_{3} \\
T=\frac{1}{2} M_{A}\left(\dot{x}_{1}^{2}+\dot{y}_{1}^{2}+\dot{z}_{1}^{2}\right)+\frac{1}{2} M_{b}\left(\dot{x}_{2}^{2}+\dot{y}_{2}^{2}+\dot{z}_{2}^{2}\right)+\frac{1}{2} M_{B}\left(\dot{x}_{3}^{2}+\dot{y}_{3}^{2}+\dot{z}_{3}^{2}\right) .
\end{gathered}
$$

Using the coordinates listed in section 2.3 .1 in eq (6), employing small angle approximations, and keeping only the significant terms, yields the result

$$
\begin{aligned}
T & =\frac{1}{2} M_{A}\left[L_{A}^{2}\left(\dot{\beta}_{y}\right)^{2}+L_{\mathrm{cm}}^{2}\left(\dot{\gamma}_{x}\right)^{2}-2 L_{\mathrm{cm}} \rho_{/}\left(\dot{\gamma}_{x}\right)^{2}\right. \\
& \left.+2 L_{A} L_{\mathrm{cm}} \gamma_{x} \dot{\gamma}_{x} \dot{\beta}_{y}\right]+\frac{1}{2} M_{b} \ell_{b}^{2}\left(\dot{\beta}_{y}\right)^{2} \\
& +\frac{1}{2} M_{B}\left[L_{B}^{2}+\ell_{B}^{2}\right]\left(\dot{\beta}_{y}\right)^{2} \\
& 2.7 .2 \gamma_{r} \text { and } \beta_{r} \text { Motions }
\end{aligned}
$$

When the coordinates listed in section 2.3.2 are used in eq (6), the kinetic energy expression becomes

$$
\begin{aligned}
T & =\frac{1}{2} M_{A}\left[L_{A}^{2}\left(\dot{\beta}_{y}\right)^{2}+L_{\mathrm{cm}}^{2}\left(\dot{\gamma}_{y}\right)^{2}-2 L_{\mathrm{cm}} \rho_{L}\left(\dot{\gamma}_{y}\right)^{2}\right. \\
& \left.+2 L_{A} L_{\mathrm{cm}}\left(\gamma_{y}+\beta_{y}\right) \dot{\gamma}_{y} \dot{\beta}_{y}+2 L_{\mathrm{cm}}\left(\rho_{C}-\rho_{L}\right) \dot{\gamma}_{y} \dot{\beta}_{y}\right] \\
& +\frac{1}{2} M_{b} \ell_{b}^{2}\left(\dot{\beta}_{y}\right)^{2}+\frac{1}{2} M_{B}\left[L_{B}^{2}+\ell_{B}^{2}\right]\left(\dot{\beta}_{y}\right)^{2}
\end{aligned}
$$

\subsection{Rayleigh's Dissipation Function}

We will assume that if the balance beam is mechanically or electronically damped, that the damping force is proportional to the velocity. Let the damping occur at point 4 of figure 1. Then for small angles, the Rayleigh dissipation function $[3], F$, is

$$
F=\frac{1}{2} \alpha \dot{z}_{4}^{2}=\frac{1}{2} \alpha \mathscr{\Psi}_{B}^{2}\left(\dot{\beta}_{y}\right)^{2},
$$

and the damping force, $F_{\alpha}$, is

$$
F_{\alpha}=-\frac{\partial F}{\partial v_{x_{a}}}=-\alpha \mathscr{L}_{B} \dot{\beta}_{y} .
$$




\subsection{D'Alembert's Principle}

\section{$2.9 .1 \gamma_{x}$ and $\beta_{x}$ Motions}

D'Alemberts' Principle $[3,4]$ for the equation of motion of the balance beam about the center knife may be written:

$$
\frac{d}{d t}\left(\frac{\partial T}{\partial \dot{\beta}_{y}}\right)-\frac{\partial T}{\partial \beta_{y}}+\frac{\partial F}{\partial \dot{\beta}_{y}}=Q_{\beta_{y}}
$$

Substituting the expressions for $Q_{\beta}, T$ and $F$ given by eqs (3), (4), (7), and (9) into (11), and replacing the point masses of figure 1 with distributed masses, yields the result

$$
\left[I_{C}\right] \ddot{\beta}_{y}+\left[\alpha_{B}^{2}\right] \dot{\beta}_{y}+\kappa \beta_{y}-\left[M_{B} g L_{B}-M_{A} g L_{A}\right]=-\left[M_{A} L_{A} L_{\mathrm{cm}}\right]\left(\dot{\gamma}_{x}\right)^{2}-\left[M_{A} L_{A} L_{\mathrm{cm}}\right] \gamma_{x} \dot{\gamma}_{x}
$$

$I_{c}$ is the moment of inertia of the balance about point $C$ of figure 1 when $\beta_{y}=0$; i.e.,

$$
\begin{aligned}
& I_{C}=I_{1}+I_{2}+I_{3} \\
& I_{C}=\left[M_{A} L_{A}^{2}\right]+\left[\left(I_{b}\right)_{\mathrm{cm}}+M_{b} \ell_{b}^{2}\right]+\left[\left(I_{B}\right)_{\mathrm{cm}}+M_{B}\left(L_{B}^{2}+\ell_{B}^{2}\right)\right],
\end{aligned}
$$

where $\left(I_{b}\right)_{\mathrm{cm}}$ and $\left(I_{B}\right)_{\mathrm{cm}}$ are the respective moments of inertia of the balance beam and the counterweight about the $\mathrm{x}$ axis through the center of mass, points 2 and 3 of figure 1 . The $M_{b} \ell_{b}^{2}$ and $M_{B}\left(L_{B}^{2}+\ell_{B}^{2}\right)$ terms result from the parallel axis theorem. The combination of suspension system plus load acts as a point mass-as far as the center knife is concerned-because of the crossed knives at point $P$.

Equation (12) has the form

$$
b \ddot{\beta}_{y}+c \dot{\beta}_{y}+\kappa \beta_{y}-d=-e\left(\dot{\gamma}_{x}\right)^{2}-e \gamma_{x} \hat{\gamma}_{x}
$$

Appendix A lists all algebraic substitutions used in the derivations, such as those for the coefficients $b, c$, $\kappa, d$ and $e$ of (14).

The r.h.s. terms of (12) and (14) can be explained by considering the torques about the center knife due to the radial and tangential accelerations of the suspension system-load mass combination about point $\left(P^{\prime}\right)$ as shown in figure 5 . The torque $\Gamma_{C}^{\prime}$ is

$$
\begin{aligned}
\Gamma_{C}^{\prime} & =\left(M_{A} a_{R} \cos \gamma_{x}\right)\left(-L_{A} \cos \beta_{y}-\rho_{L} \sin \beta_{y}-\rho_{C} \beta_{y}\right) \\
& +\left(M_{A} a_{T} \sin \gamma_{x}\right)\left(-L_{A} \cos \beta_{y}-\rho_{L} \sin \beta_{y}-\rho_{C} \beta_{y}\right)
\end{aligned}
$$

where $a_{R}=L_{\mathrm{cm}}\left(\dot{\gamma}_{x}\right)^{2}$ and $a_{T}=L_{\mathrm{cm}} \hat{\gamma}_{x}$. Note that the torque $\Gamma_{C}^{\prime}$ is about the pivot point $(0)$ of the center knife rather than about the origin $(C)$, or about the contact point $\left(C^{\prime}\right)$; (see fig. 2). For small angles, eq (15) reduces to

$$
\Gamma_{C}^{\prime}=-\left[M_{A} L_{A} L_{\mathrm{cm}}\right]\left(\dot{\gamma}_{x}\right)^{2}-\left[M_{A} L_{A} L_{\mathrm{cm}}\right] \gamma_{x} \dot{\gamma}_{x}=-e\left(\dot{\gamma}_{x}\right)^{2}-e \gamma_{x} \dot{\gamma}_{x}
$$

thus accounting for the r.h.s. terms of (12) and (14) by using Newtonian mechanics.

$$
2.9 .2 \gamma_{r} \text { and } \beta \text {, Motions }
$$

Substituting the expressions for $Q_{\beta}, T$ and $F$ given by eqs (3), (4), (8) and (9) into (11), and using distributed masses for the balance, yields the result 
$\left[I_{C}\right] \ddot{\beta}_{y}+\left[\alpha_{B}^{2}\right] \dot{\beta}_{y}+\kappa \beta_{y}-\left[M_{B g} L_{B}-M_{A} g L_{A}\right]$

$$
=-\left[M_{A} L_{A} L_{\mathrm{cm}}\right]\left(\dot{\gamma}_{y}\right)^{2}-\left[M_{A} L_{A} L_{\mathrm{cm}}\right]\left(\gamma_{y}+\beta_{y}\right) \psi_{y}-\left[M_{A} L_{\mathrm{cm}}\left(\rho_{C}-\rho_{L}\right)\right] \psi_{y},
$$

which has the form

$$
b \ddot{\beta}_{y}+c \dot{\beta}_{y}+\kappa \beta_{y}-d=-e\left(\dot{\gamma}_{y}\right)^{2}-e\left(\gamma_{y}+\beta_{y}\right) \gamma_{y}-f \gamma_{y} .
$$

Referring to figure 5, the torques about the pivot point (0) of the knife due to the radial and tangential accelerations of the suspension system-load mass combination are

$$
\begin{aligned}
\Gamma_{C} & =\left(M_{A} a_{R} \cos \gamma_{y}\right)\left(-L_{A} \cos \beta_{y}-\rho_{L} \sin \beta_{y}-\rho_{L} \sin \gamma_{y}-\rho_{C} \beta_{y}\right) \\
& +\left(M_{A} a_{R} \sin \gamma_{y}\right)\left[-L_{A} \sin \beta_{y}-\rho_{L} \cos \gamma_{y}+\rho_{L} \cos \beta_{y}-\rho_{C}\left(1-\cos \beta_{y}\right)-\rho_{C}\right] \\
& +\left(M_{A} a_{T} \cos \gamma_{y}\right)\left[-L_{A} \sin \beta_{y}-\rho_{L} \cos \gamma_{y}+\rho_{L} \cos \beta_{y}-\rho_{C}\left(1-\cos \beta_{y}\right)-\rho_{C}\right] \\
& +\left(M_{A} a_{T} \sin \gamma_{y}\right)\left(-\dot{L}_{A} \cos \beta_{y}-\rho_{L} \sin \beta_{y}-\rho_{L} \sin \gamma_{y}-\rho_{C} \beta_{y}\right)-\left(M_{A} a_{T}\right)\left(-\rho_{L}\right),
\end{aligned}
$$

where $a_{R}=L_{\mathrm{cm}}\left(\dot{\gamma}_{y}\right)^{2}$ and $a_{T}=L_{\mathrm{cm}} \psi_{\gamma}$. The last term in (18) results from a torque about the center of curvature of the load knife due to an action-reaction force $M_{A} a_{T}$ applied to the non-slipping contact point $\left(P^{\prime}\right)$; this torque is transmitted to point (0) because the balance beam is rigid.

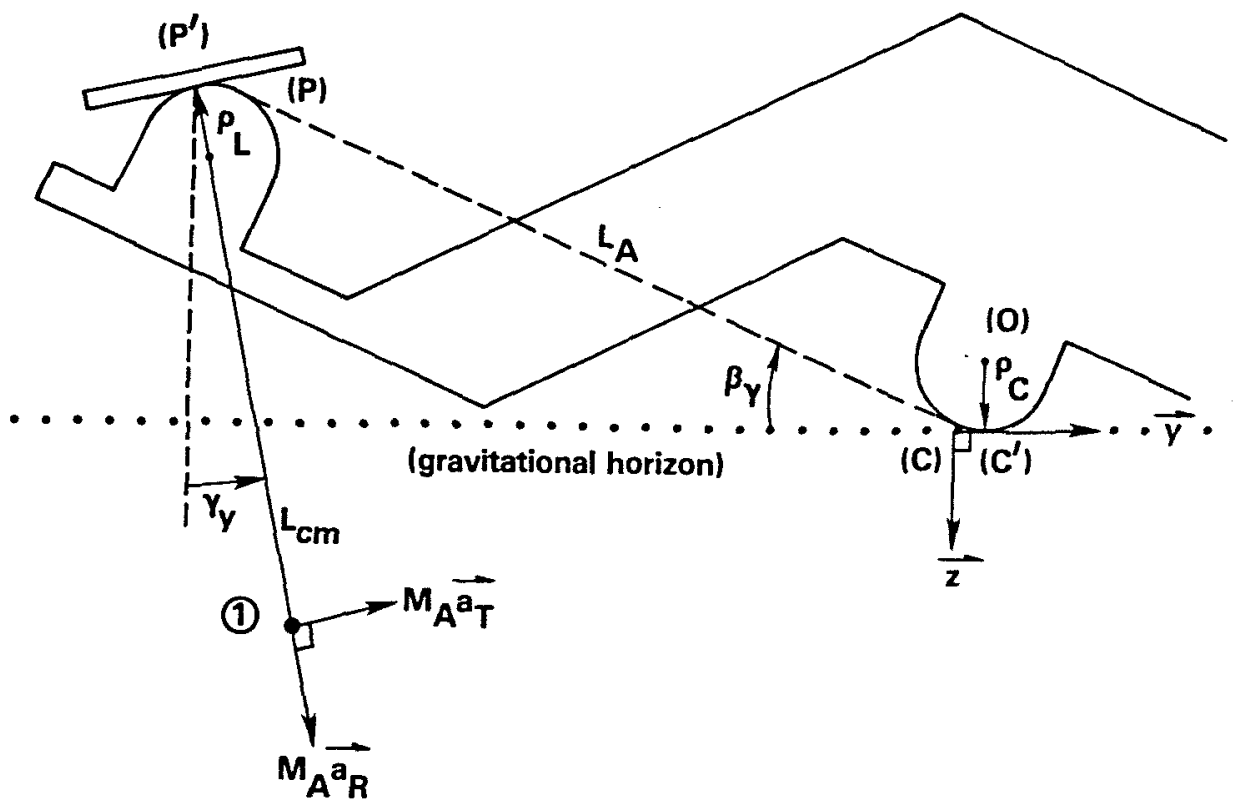

Ficure 5. Dynamically induced forces on the swinging pendulum. These forces produce torques about the pirot axis of the center knife. This axis, which is in the $x$ direction, passes through the center of curvature of the center knife at point $(0)$.

For small angles, eq (18) becomes

$$
\begin{aligned}
\Gamma_{C} & =-\left[M_{A} L_{A} L_{\mathrm{cm}}\right]\left(\dot{\gamma}_{y}\right)^{2}-\left[M_{A} L_{A} L_{\mathrm{cm}}\right]\left(\gamma_{y}+\beta_{y}\right) \psi_{y}-\left[M_{A} L_{\mathrm{cm}}\left(\rho_{C}-\rho_{L}\right)\right] \psi_{y} \\
& =-e\left(\dot{\gamma}_{y}\right)^{2}-e\left(\gamma_{y}+\beta_{y}\right) \psi_{y}-f \gamma_{y},
\end{aligned}
$$

thus accounting for the r.h.s. terms of (17). 
The $-\left[M_{A} L_{A} L_{\mathrm{cm}}\right] \beta_{y} \psi_{y}=-\mathrm{e} \beta_{y} \psi_{y}$ term of (16) (which is due to $M_{A}$ times the horizontal component of the tangential acceleration times the change in height from gravitational horizon of the load end of the balance beam) will later be seen to have a special significance. We will refer to this quantity (divided by $\boldsymbol{l}_{C}$ ) as the Mathieu term. (There is also a Mathieu term contribution due to the horizontal component of the radial acceleration; but it is negligible for small angles.) The quantity $\left[M_{A} L_{\mathrm{cm}}\left(\rho_{C}-\rho_{L}\right) / I_{C} \psi_{Y}\right.$ will later be referred to as the knife-edges term.

\subsection{Physical Pendulum}

\subsection{1 $\gamma_{k}$ and $\beta_{y}$ Motions}

We assume that the motion of the suspension system-load combination is that of a physical pendulum, and arbitrarily choose $\gamma_{x}$ to be a minimum at time $t=0$ and to have an amplitude $\bar{\gamma}_{x} ;$ i.e.,

$$
\gamma_{x}=-\gamma_{x} \cos \omega_{\gamma_{x}} t
$$

where

$$
\begin{gathered}
\omega_{\gamma_{x}}^{2}=\frac{\left[M_{A} g L_{\mathrm{cm}}\right]}{I_{P}^{\prime}}, \\
I_{P}^{\prime}=\left(I_{S}^{\prime}\right)_{\mathrm{cm}}+M_{A} L_{\mathrm{cm}}^{2},
\end{gathered}
$$

and

$$
\omega_{\gamma_{x}}=\frac{2 \pi}{P_{\gamma_{x}}}
$$

$I_{P}^{\prime}$ is the moment of inertia of the physical pendulum about the y axis at point $(P)$ of figure $1,\left(I_{S}^{\prime}\right)_{\mathrm{cm}}$ is the moment of inertia of the suspension system-load mass combination about the $y$ axis through the center of mass point 1 of figure 1 , and $\boldsymbol{P}_{\boldsymbol{\gamma}_{\boldsymbol{x}}}$ is the pendulum period.

The higher order modulation terms have been ignored in (19) because they do not significantly affect the balance beam motion. They could be readily obtained by considering the equations of motion about point $(P)$ of figure 1 .

\subsection{2 $\gamma$, and $\beta$, Motions}

Analogous to section 2.10.1:

$$
\begin{gathered}
\gamma_{y}=-\tilde{\gamma}_{y} \cos \omega_{\gamma_{v}} t, \\
\omega_{\gamma_{v}}^{2}=\frac{\left[M_{A} g L_{\mathrm{cm}}\right]}{I_{P}}, \\
I_{P}=\left(I_{S}\right)_{\mathrm{cm}}+M_{A} L_{\mathrm{cm}}^{2},
\end{gathered}
$$

and

$$
\omega_{\gamma_{\gamma}}=\frac{2 \pi}{P_{\gamma_{\nu}}} .
$$

$I_{P}$ and $\left(I_{s}\right)_{\mathrm{cm}}$ are the moments of inertia about the $x$ axis through points $(\mathrm{P})$ and 1 , respectively of figure 1. 


\section{Solution for $\gamma_{x}$ and $\beta_{y}$ Motions}

The equation of motion can now be expressed in final form by substituting eq (19) into (14), and by using the identity $\cos ^{2} \psi-\sin ^{2} \psi=\cos 2 \psi$; it is

$$
\ddot{\beta}_{y}+\frac{c}{b} \dot{\beta}_{y}+\frac{\kappa}{b} \beta_{y}-\frac{d}{b}=\frac{e}{b} \tilde{\gamma}_{x}^{2} \omega_{\gamma_{x}}^{2} \cos 2 \omega_{\gamma_{x}} t
$$

The general solution of (27) will consist of the solutions to the homogeneous equation (where the r.h.s. of (27) is zero), plus a particular solution.

The homogeneous solution to this equation is easily obtained by standard techniques. The particular solution can be found by writing (27) in the form

$$
\ddot{\beta}_{y}+\bar{u} \dot{\beta}_{y}+v^{2} \beta_{y}-\frac{d}{b}=w \cos k t
$$

and by assuming that the solution is

$$
\beta_{y}=D \cos k t+E \sin k t+\hat{\beta}_{y}
$$

which yields the results

$$
D=\frac{\bar{u} w k}{\left[\left(v^{2}-k^{2}\right)^{2}+\bar{u}^{2} k^{2}\right]}
$$

and

$$
E=\frac{w\left(v^{2}-k^{2}\right)}{\left[\left(v^{2}-k^{2}\right)^{2}+\bar{u}^{2} k^{2}\right]}
$$

The general solution of (27) is therefore

$$
\begin{aligned}
& \beta_{y}=\left\{e^{-\frac{a t}{2 b}}\left[A \exp \sqrt{\omega_{\beta_{y}^{\prime}}^{\prime} t}+B \exp -\sqrt{-\omega_{\beta_{v}^{\prime 2}}^{\prime 2} t}\right]\right. \\
& \text { or }(A+B t) e^{-\frac{d}{2 b}} \text { or } \\
& \left.e^{-\frac{a t}{2 b}}\left[A \cos \omega_{\beta,}^{\prime} t+B \sin \omega_{\beta, t}^{\prime} t\right]\right\} \\
& +\hat{\beta}_{y}+\frac{e}{b} \gamma_{z}^{2} \omega_{\gamma_{y}}^{2} \frac{\left(\omega_{\beta_{2}}^{2}-4 \omega_{\gamma_{2}}^{2}\right) \cos 2 \omega_{\gamma_{2}} t}{\left[\left(\omega_{\beta_{y}}^{2}-4 \omega_{\gamma_{2}}^{2}\right)^{2}+4 \frac{c^{2}}{b^{2}} \omega_{\gamma_{x}}^{2}\right]} \\
& +2 \frac{c e}{b^{2}} \gamma_{x}^{2} \omega_{\gamma_{x}}^{3} \frac{\sin 2 \omega_{\gamma_{x}} t}{\left[\left(\omega_{\beta_{y}}^{2}-4 \omega_{\gamma_{x}}^{2}\right)^{2}+4 \frac{c^{2}}{b^{2}} \omega_{\gamma_{x}}^{2}\right]} \text {, }
\end{aligned}
$$

where

$$
\begin{aligned}
& \omega_{\beta,}^{2}=\kappa / b, \\
& \omega_{\beta,}^{\prime 2}=\frac{\kappa}{b}-\frac{c^{2}}{4 b^{2}},
\end{aligned}
$$


and

$$
\hat{\beta}_{y}=\frac{d}{\kappa}=\frac{\left[M_{B g} g L_{B}-M_{A} g L_{A}\right]}{\left[M_{A} g\left(\rho_{L}+\rho_{C}\right)+M_{b} g\left(\ell_{b}+\rho_{C}\right)+M_{B} g\left(\ell_{B}+\rho_{C}\right)\right]}
$$

The choice of terms in eq (28) arises because the balance may be overdamped, critically damped, or underdamped depending on whether $c^{2} / 4 b$ is greater than, equal to, or less than $K$. For all damping conditions the motion of the balance beam about the equilibrium angle $\hat{\beta}_{y}$ is modulated at an angular frequency which is twice that of the natural angular frequency $\omega_{\gamma_{x}}$ of the physical pendulum. This modulation effect is due to the radial and tangential accelerations of the suspension system-load mass combination.

\section{Solution for $\gamma_{y}$ and $\beta_{y}$ Motions}

\subsection{Equation of Motion}

The equation of motion is obtained by substituting eq (23) into (17), and by using the identity $\cos ^{2} \psi-$ $\sin ^{2} \psi=\cos 2 \psi:$

$$
\frac{d^{2} \beta_{y}}{d t^{2}}+\frac{c}{b} \frac{d \beta_{y}}{d t}+\left(\frac{\kappa}{b}+\frac{e}{b} \tilde{\gamma}_{y} \omega_{\gamma_{y}}^{2} \cos \omega_{\gamma_{y}} t\right) \beta_{y}=\frac{d}{b}+\frac{e}{b} \gamma_{y}^{2} \omega_{\gamma_{y}}^{2} \cos 2 \omega_{\gamma_{y}} t-\frac{f}{b} \bar{\gamma}_{y} \omega_{\gamma_{y}}^{2} \cos \omega_{\gamma} t .
$$

The $\left(\frac{e}{b} \gamma_{y} \omega_{\gamma}^{2}, \cos \omega_{\gamma_{y}}\right) \beta_{y}$ term of eq (32)-which is the Mathieu term referred to in section 2.9.2-complicates the problem; yet it cannot, in general, be neglected when considering the dynamical effects of precision balances. Therefore, (32) will be converted to a more tractable form.

First, we change from the time variable, $t$, to the dimensionless variable $z$ by making the substitution

$$
\omega_{\gamma_{y}} t=2 z \text {. }
$$

Equation (32) thus becomes

$$
\begin{aligned}
\frac{d^{2} \beta_{y}}{d z^{2}}+2\left[\frac{c}{b \omega_{\gamma_{y}}}\right] \frac{d \beta_{y}}{d z}+\left(\left[4 \frac{\omega_{\mathrm{B}_{z}}^{2}}{\omega_{\gamma_{y}}^{2}}\right]-2\left[\frac{-2 e}{b} \gamma_{y}\right] \cos 2 z\right) \beta_{y} \\
=\left[\frac{4}{\omega_{\gamma_{y}}^{2}} \frac{d}{b}\right]+\left[4 \frac{e}{b} \gamma_{y}^{2}\right] \cos 2 \omega_{\gamma_{y}} t-\left[4 \frac{f}{b} \gamma_{y}\right] \cos \omega_{\gamma_{y}} t,
\end{aligned}
$$

or

$$
\frac{d^{2} \beta_{y}}{d z^{2}}+2 \bar{k} \frac{d \beta_{y}}{d z}+(\bar{a}-2 q \cos 2 z) \beta_{y}=h+\ell \cos 4 z-m \cos 2 z=\bar{f}(z) .
$$

We then replace $\beta_{y}$ with the quantity [10]

$$
\beta_{y}=e^{-i z} u(z)
$$

thus obtaining the equation of motion in final form:

$$
\frac{d^{2} u}{d z^{2}}+(a-2 q \cos 2 z) u=e^{i z} \bar{f}(z)
$$


where

$$
a=\bar{a}-\bar{k}^{2}=\frac{4}{\omega_{\gamma,}^{2}}\left[\frac{\kappa}{b}-\frac{c^{2}}{4 b^{2}}\right]
$$

The quantity $a$ is negative if the balance beam is overdamped, zero if critically damped, and positive if underdamped; whereas $q$ is always a negative number. The quantities $z, \bar{f}(z), u(z), \bar{k}, \bar{a}, a, q, h, \ell$, and $m$ are all dimensionless. The general solution of (37) consists of the solutions to the homogeneous equation, plus a particular solution.

\subsection{Homogeneous Solutions}

The homogeneous equation

$$
u^{\prime \prime}+(a-2 q \cos 2 z) u=0
$$

has the canonical form of Mathieu's equation $[9,10]$. The reader is referred to reference [10] for an excellent account of how to obtain solutions to this equation.

Figure 6 shows an isostability diagram [10] of $v$ for possible solutions to eq (39). We are only interested in underdamped or critically damped solutions because laboratory analytical balances are seldom designed to operate in the overdamped mode; therefore $1>>a \geqslant 0$. Also, the quantity $g=-2 M_{A} L_{A} L_{\mathrm{cm}} \bar{\gamma}_{y} / I_{C}$ is always a very small negative real number for precision balances; so $-1<<q<0$. The region of interest for these values of $a$ and $q$ is indicated by an arrow in figure 6. Clearly, the solutions of (39) cannot be "simple" Mathieu functions of the sine or cosine types $s\left(e_{1}\right), c\left(e_{0}\right)$ or $c\left(e_{1}\right)[9,10]$, but instead are more complicated functions.

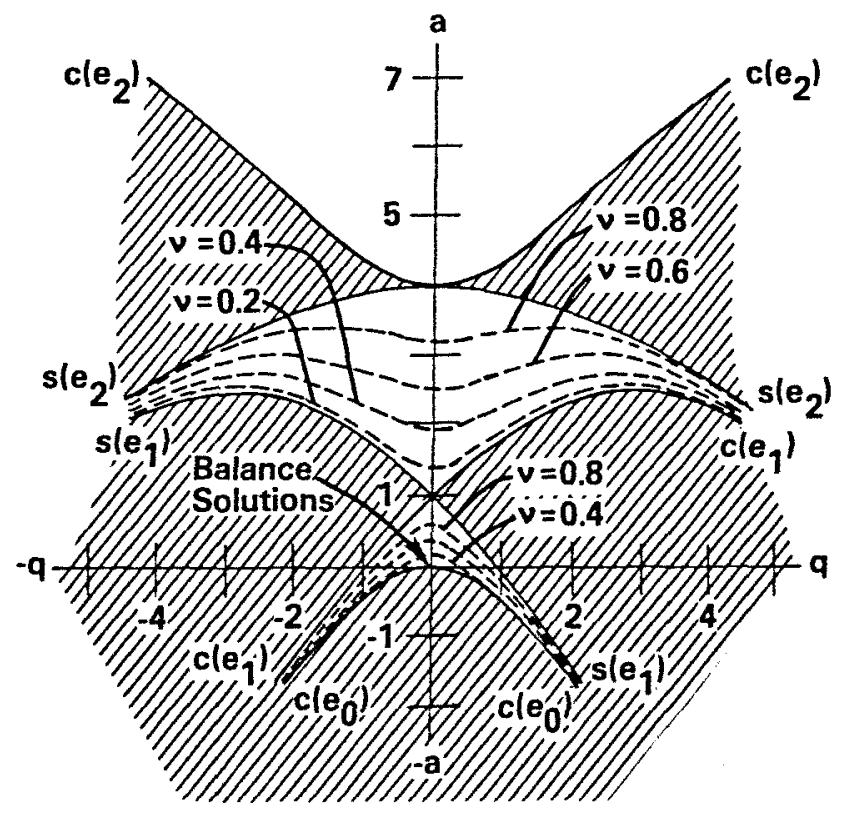

Ficure 6. An isostability diagram of $v$ for possible solutions of Mathieu's equation given by (39). The arrow points to the region of interest for precision balances; i.e. $1>>a \geqslant 0$ and $-1<<q<0$. Solutions in the shaded region are unstable. This does not mean that an overdamped balance $(a<0)$ is mechanically unstable because $u(z)$ is multiplied by the factor $e^{-i x}$ in the solution for $\beta$. 
Since $q$ is small, we guess that the solution is of the Floquet form [9]

$$
u=e^{i v q}-\frac{q e^{i(v+2) z}}{4(v+1)}+\frac{q e^{i(\nu-2) z}}{4(v-1)}
$$

where $\nu$ is not an integer; (i.e., $u(z)$ is not a Mathieu function). For small values of $a, v$ is defined by [9]

$$
\begin{aligned}
\cos v \pi= & \left(1-\frac{a \pi^{2}}{2}+\frac{a^{2} \pi^{4}}{24}+\cdots\right)-\frac{q^{2} \pi^{2}}{4}\left[1+a\left(1-\frac{\pi^{2}}{6}\right)+\cdots\right] \\
& +q^{4}\left(\frac{\pi^{4}}{96}-\frac{25 \pi^{2}}{256}+\cdots\right)+\cdots
\end{aligned}
$$

We then use the identity $e^{i \theta}=\cos \theta+i \sin \theta$ in (40) to obtain the two independent solutions of eq (39); they are

$$
u_{1}=\cos v z-\bar{q} \cos v z \cos 2 z-\nu \bar{q} \sin v z \sin 2 z
$$

and

$$
u_{2}=\sin \nu z-\bar{q} \sin \nu z \cos 2 z+\nu \bar{q} \cos \nu z \sin 2 z
$$

where terms of order $\vec{q}^{2}$ have been neglected, and

$$
\begin{aligned}
& \bar{q}=\frac{q}{2\left(1-v^{2}\right)}, \\
& \nu \approx \sqrt{a} \quad(\text { for } a>0),
\end{aligned}
$$

or

$$
v \approx \frac{q}{\sqrt{2}} \quad(\text { for } a=0)
$$

The homogeneous solution of (39) is therefore

$$
A u_{1}(z)+B u_{2}(z)
$$

\subsection{General Solution}

\subsubsection{Particular Solution}

We must next find a particular solution, $u_{p}(z)$, of

$$
u^{\prime \prime}+(a-2 q \cos 2 z) u=e^{k z} \bar{f}(z)
$$

which has the form of Hill's equation [10]. The solution is obtained from the expression

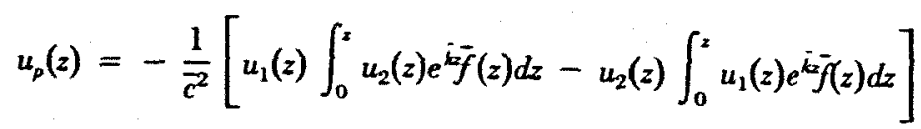

where $u_{1}$ and $u_{2}$ are given by eqs (41) and (42), $\bar{f}(z)=h+\ell \cos 4 z-m \cos 2 z$, and 


$$
\vec{c}^{2}=u_{1}(z) u_{2}^{\prime}(z)-u_{2}(z) u_{1}^{\prime}(z)=v
$$

The evaluation of eq (47) is straightforward, but very lengthy; the result is included in the next subsection.

\subsubsection{Complete Solution}

The general solution to eq (35), using (36), (45), (47), and (48), has the form

$$
\beta_{y}=e^{-i_{z}} u(z)=e^{-\bar{k}_{z}}\left[A u_{1}(z)+B u_{2}(z)\right]-\frac{e^{-i z}}{\nu}\left[u_{1}(z) \int_{0}^{z} u_{2}(z) e^{-\bar{k} z} f(z) d z-u_{2}(z) \int_{0}^{z} u_{1}(z) e^{k_{z}} f(z) d z\right]
$$

which, when using (41) and (42) is

$$
\begin{aligned}
& \beta_{y}=A e^{-\bar{k}}[\cos \nu z-\bar{q} \cos v z \cos 2 z-\nu \bar{q} \sin \nu z \sin 2 z] \\
& +B e^{-k_{z}}[\sin v z-\bar{q} \sin v z \cos 2 z+\nu \bar{q} \cos \nu z \sin 2 z] \\
& -\frac{1}{\nu\left[\bar{k}^{2}+v^{2}\right]}\left\{\left(h+\frac{\bar{q}}{2} m\right)\left[-v+e^{-i z}(\nu \cos v z+\bar{k} \sin v z)\right]\right. \\
& -(\bar{q} h)\left[-v \cos 2 z+e^{-i z}(\nu \cos v z \cos 2 z+\bar{k} \sin v z \cos 2 z)\right] \\
& \left.-(\nu \bar{q} h)\left[\bar{k} \sin 2 z+e^{-\bar{k} z}(\nu \sin \nu z \sin 2 z+\bar{k} \cos \nu z \sin 2 z)\right]\right\} \\
& -\frac{1}{2 \nu\left[\bar{k}^{2}+(\nu-2)^{2}\right]}\left\{\left(-m-\bar{q} h-\frac{\bar{q}}{2} \ell-v \bar{q} h+\frac{\nu \bar{q}}{2} \ell\right)[-\bar{k} \sin 2 z\right. \\
& \left.-(\nu-2) \cos 2 z+e^{-i z}((\nu-2) \cos v z+\bar{k} \sin v z)\right] \\
& +(\bar{q} m)\left[-\bar{k} \sin 2 z \cos 2 z-(\nu-2) \cos ^{2} 2 z+e^{-\bar{k}}((\nu-2) \cos v z \cos 2 z+\bar{k} \sin v z \cos 2 z)\right] \\
& \left.+(\nu \bar{q} m)\left[-(\nu-2) \sin ^{2} 2 z+\bar{k} \sin 2 z \cos 2 z+e^{-\bar{k} z}((v-2) \sin v z \sin 2 z-\bar{k} \cos v z \sin 2 z)\right]\right\} \\
& -\frac{1}{2 \nu\left[\bar{k}^{2}+(\nu+2)^{2}\right]}\left\{\left(-m-\bar{q} h-\frac{\bar{q}}{2} \ell+\nu \bar{q} h-v \frac{\bar{q}}{2} \ell\right)[\bar{k} \sin 2 z\right. \\
& \left.-(\nu+2) \cos 2 z+e^{-i x}((\nu+2) \cos v z+i \sin \nu z)\right] \\
& +(\bar{q} m)\left[\bar{k} \sin 2 z \cos 2 z-(\nu+2) \cos ^{2} 2 z+e^{-\bar{k}}((\nu+2) \cos \nu z \cos 2 z+\bar{k} \sin \nu z \cos 2 z)\right] \\
& \left.+(\nu \bar{q} m)\left[\bar{k} \sin 2 z \cos 2 z+(\nu+2) \sin ^{2} 2 z+e^{-\bar{k} z}((\nu+2) \sin v z \sin 2 z-\bar{k} \cos v z \sin 2 z)\right]\right\} \\
& -\frac{1}{2 v\left(\bar{k}^{2}+(\nu-4)^{2}\right]}\left\{\left(\ell+\frac{\bar{q}}{2} m+v \frac{\bar{q}}{2} m\right)[-\bar{k} \sin 4 z-(\nu-4) \cos 4 z\right. \\
& \left.+e^{-\bar{k}}((\nu-4) \cos v z+\bar{k} \sin v z)\right] \\
& -(\bar{q} \ell)\left[-\bar{k} \cos 2 z \sin 4 z-(\nu-4) \cos 2 z \cos 4 z+e^{-\bar{k}}((\nu-4) \cos v z \cos 2 z+\bar{k} \sin v z \cos 2 z)\right] \\
& \left.-(\nu \bar{q} \ell)\left[\bar{k} \sin 2 z \cos 4 z-(\nu-4) \sin 2 z \sin 4 z+e^{-\bar{k} z}((\nu-4) \sin \nu z \sin 2 z-\bar{k} \cos \nu z \sin 2 z)\right]\right\} \\
& -\frac{1}{2 v\left[\bar{k}^{2}+(v+4)^{2}\right]}\left\{\left(\ell+\frac{\vec{q}}{2} m-v \frac{\bar{q}}{2} m\right)[\vec{k} \sin 4 z-(v+4) \cos 4 z\right. \\
& \left.+e^{-i z}((\nu+4) \cos v z+\bar{k} \sin v z)\right]
\end{aligned}
$$




$$
\begin{aligned}
& -(\bar{q} \ell)\left[\bar{k} \cos 2 z \sin 4 z-(\nu+4) \cos 2 z \cos 4 z+e^{-\bar{k} z}((\nu+4) \cos v z \cos 2 z+\bar{k} \sin v z \cos 2 z)\right] \\
& \left.-(v \bar{q} \ell)\left[\bar{k} \sin 2 z \cos 4 z+(v+4) \sin 2 z \sin 4 z+e^{-\bar{k} z}((v+4) \sin v z \sin 2 z-\bar{k} \cos v z \sin 2 z)\right]\right\} \\
& -\frac{1}{2 v\left[\bar{k}^{2}+(v-6)^{2}\right]}\left\{\left(-\frac{\bar{q}}{2} \ell-v \frac{\bar{q}}{2} \ell\right)[-\bar{k} \sin 6 z-(v-6) \cos 6 z\right. \\
& \left.\left.+e^{-\bar{k} z}((\nu-6) \cos v z+\bar{k} \sin v z)\right]\right\} \\
& -\frac{1}{2 v\left[\bar{h}^{2}+(v+6)^{2}\right]}\left\{\left(-\frac{\bar{q}}{2} \ell+v \frac{\bar{q}}{2} \ell\right)[\bar{k} \sin 6 z-(\nu+6) \cos 6 z\right. \\
& \left.\left.+e^{-k z}((\nu+6) \cos v z+\bar{k} \sin v z)\right]\right\} \text {. }
\end{aligned}
$$

This is the exact solution (in reduced notation) for small angle $\gamma_{y}$ and $\beta_{y}$ motions. It is obviously much more complicated than the solution for small angle $\gamma_{x}$ and $\beta_{y}$ motions given by eq (28).

It requires too many printed pages to provide an expanded form of eq (50) written in the notation of (28). (There are 74 terms in that notation.) However, the reader can readily transform any term of $(50)$ into that notation with the aid of Appendices A and B. Equation (50) is provided so that readers seeking solutions to problems similar to our own may be spared the considerable tedium of its derivation and so that the terms neglected in the sequel may be recovered by the interested reader.

\section{Special Cases}

\subsection{A Freely-Swinging Balance}

In a completely undamped balance, $\bar{k}=c=0$, and $\omega_{\beta,}^{\prime}=\omega_{\beta y} ;$ so eq (28) becomes

$$
\beta_{y}=A \cos \omega_{\beta_{y}} t+B \sin \omega_{\beta_{y}} t+\hat{\beta}_{y}+\frac{e}{b} \hat{\gamma}_{x}^{2} \frac{\omega_{\gamma_{x}}^{2}}{\left(\omega_{\beta_{y}}^{2}-4 \omega_{\gamma_{x}}^{2}\right)} \cos 2 \omega_{\gamma_{x}} t
$$

Using Appendices A and B, and keeping only the largest terms, (50) becomes

$$
\begin{aligned}
& \beta_{y}=A \cos \omega_{\beta_{y}} t+B \sin \omega_{\beta_{y}} t+\left[\hat{\beta}_{y}-\frac{e f}{2 b^{2}} \tilde{\gamma}_{y}^{2} \frac{\omega_{\gamma_{y}}^{4}}{\omega_{\beta_{y}}^{2}\left(\omega_{\gamma_{y}}^{2}-4 \omega_{\beta_{y}}^{2}\right)}\right]
\end{aligned}
$$

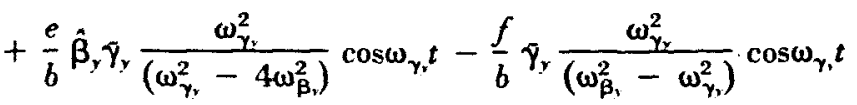

$$
\begin{aligned}
& +\frac{e}{b} \gamma_{y}^{2} \frac{\omega_{\gamma_{\gamma}}^{2}}{\left(\omega_{\beta_{\gamma}}^{2}-4 \omega_{\gamma_{\gamma}}^{2}\right)} \cos 2 \omega_{\gamma_{\gamma}} t+\frac{e^{2}}{2 b^{2}} \gamma_{y}^{3} \frac{\omega_{\gamma_{\gamma}}^{4}}{\left(\omega_{\gamma_{\gamma}}^{2}-4 \omega_{\beta_{\gamma}}^{2}\right)\left(\omega_{\beta_{\gamma}}^{2}-9 \omega_{\gamma_{\gamma}}^{2}\right)} \cos 3 \omega_{\gamma_{\gamma}} t \text {, }
\end{aligned}
$$

where $b=I_{C}, e=M_{A} L_{A} L_{c \mathrm{~cm}}$ and $f=M_{A} L_{c \mathrm{~m}}\left(\rho_{C}-\rho_{L}\right)$. Only cosine modulations of the $A \cos \omega_{B_{\gamma}} t+$ $B \sin \omega_{\beta}, t$ motion appear in (51) and (52) because of the choice of the phases of the $\gamma_{x}$ and $\gamma_{y}$ motions given by eqs (19) and (23), respectively.

The $\cos 3 \omega_{\gamma_{v}} t$ term of (52) is much smaller than the $\cos 2 \omega_{\gamma_{v}} \ell$ term, and is therefore negligible. There is a systematic error term in the equilibrium angle: $-e f \tilde{\gamma}_{y}^{2} \omega_{\gamma}^{4} /\left[2 b^{2} \omega_{\beta_{y}}^{2}\left(\omega_{\gamma_{y}}^{2}-4 \omega_{\beta_{v}}^{2}\right)\right]$. This term is probably small for most precision balances and may in fact be an artifact of our initial neglect of terms in $\gamma_{y}^{2}$.

The size of the $\cos \omega_{y_{t}} t$ modulation depends upon the knife edge radii $\rho_{C}$ and $\rho_{L}$, and also upon the value of $\hat{\beta}_{y}$. The $\cos \omega_{y} t$ modulations will be at a minimum for a given $\hat{\gamma}_{y}$, when $\hat{\beta}_{y}$, is chosen (usually via an 
appropriate adjustment of $M_{A}$ ) such that the Mathieu term contribution is nearly equal and opposite to the knife-edges contribution. These two terms then nearly cancel, and one should observe pure $\cos 2 \omega_{\gamma_{y}} \bmod$ ulations. In most realistic cases, the knife-edges term can be safely ignored. (The radius of curvature of a good knife is usually less than $1 \mu \mathrm{m}$ [12].) Then the Mathieu term vanishes when the equilibrium position of the beam is parallel to the gravitational horizon $\left(\hat{\beta}_{y}=0\right)$. Note that a test for the absence of a systematic error contribution to $\hat{\beta}_{y}$ does not $a$ fortiori assure a negligible knife-edges contribution to the $\cos \omega_{\gamma_{v}} t$ modulations because the error term goes as $\psi_{y}^{2}$, whereas the modulation term depends upon $\gamma_{y}$-and is therefore much larger.

\subsection{A Damped Balance}

\subsubsection{Theoretical Predictions}

We shall now investigate the motion of a damped balance at a time $t$ which is long enough so the involving the factor $e^{-\bar{k} x}=e^{-c t / 2 b}$ become negligible; (i.e., a time such that, in the absence o the balance beam would be at rest at the equilibrium angle $\hat{\beta}_{y}$ ). Equation (31) thus becomes

$$
\begin{aligned}
\beta_{y}= & \hat{\beta}_{y}+\frac{e}{b} \tilde{\gamma}_{x}^{2} \frac{\omega_{\gamma_{x}}^{2}\left(\omega_{\beta_{v}}^{2}-4 \omega_{\gamma_{x}}^{2}\right)}{\left[\left(\omega_{\beta_{y}}^{2}-4 \omega_{\gamma_{x}}^{2}\right)^{2}+4 \frac{c^{2}}{b^{2}} \omega_{\gamma_{x}}^{2}\right]} \cos 2 \omega_{\gamma_{x}} t \\
& +\frac{2 c e}{b^{2}} \tilde{\gamma}_{x}^{2} \frac{\omega_{\gamma_{x}}^{3}}{\left[\left(\omega_{\beta_{x}}^{2}-4 \omega_{\gamma_{x}}^{2}\right)^{2}+\frac{4 c^{2}}{b^{2}} \omega_{\gamma_{x}}^{2}\right]} \sin 2 \omega_{\gamma_{x}} t
\end{aligned}
$$

and (50) becomes

$$
\begin{aligned}
& \beta_{y}=\left[\hat{\beta}_{y}-\frac{e f}{2 b^{2}} \bar{\gamma}_{y}^{2} \frac{\omega_{\gamma_{v}}^{4}}{\omega_{\beta_{,}}^{2}\left(\omega_{\gamma_{v}}^{2}-4 \omega_{\beta_{v}}^{\prime 2}\right)}\right] \\
& +\frac{e}{b} \hat{\beta}_{y} \bar{\gamma}_{y} \frac{\omega_{\gamma_{1}}^{2}}{\left(\omega_{\gamma_{y}}^{2}-4 \omega_{\beta_{y}}^{\prime 2}\right)} \cos \omega_{\gamma_{1}} t
\end{aligned}
$$

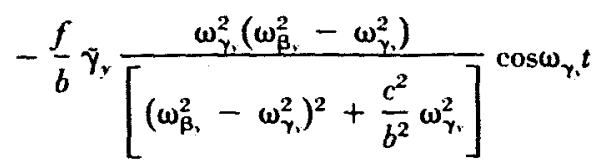

$$
\begin{aligned}
& -\frac{c e}{b^{2}} \hat{\beta}_{y} \hat{\gamma}_{y} \frac{\omega_{\gamma_{r}}}{\left(\omega_{\gamma_{,}}^{2}-4 \omega_{\beta^{\prime}}^{\prime 2}\right)} \sin \omega_{\gamma_{t} t} \\
& -\frac{c f}{b^{2}} \hat{\gamma}_{y} \frac{\omega_{\gamma_{2}}^{3}}{\left[\left(\omega_{\beta_{1}}^{2}-\omega_{\gamma_{1}}^{2}\right)^{2}+\frac{c^{2}}{b^{2}} \omega_{\gamma_{1}}^{2}\right]} \sin \omega_{\gamma_{1}} t
\end{aligned}
$$

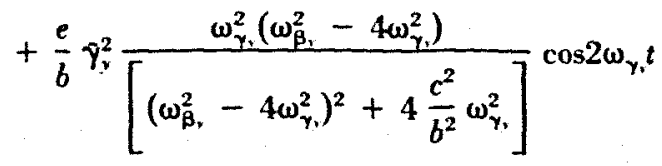

$$
\begin{aligned}
& +\frac{2 c e}{b^{2}} \gamma_{y}^{2} \frac{\omega_{\gamma_{\nu}}^{3}}{\left[\left(\omega_{\beta_{,}}^{2}-4 \omega_{\gamma_{\nu}}^{2}\right)^{2}+4 \frac{c^{2}}{b^{2}} \omega_{\gamma_{\gamma}}^{2}\right]} \sin 2 \omega_{\gamma_{y}} t
\end{aligned}
$$




$$
\begin{aligned}
& +\frac{e^{2}}{2 b^{2}} \gamma_{y}^{3} \frac{\omega_{\gamma_{y}}^{4}\left(\omega_{\beta_{\gamma}}^{2}-9 \omega_{\gamma_{\gamma}}^{2}\right)}{\left(\omega_{\gamma_{y}}^{2}-4 \omega_{\beta_{y}}^{2}\right)\left[\left(\omega_{\beta_{y}}^{2}-9 \omega_{\gamma_{y}}^{2}\right)^{2}+9 \frac{c^{2}}{b^{2}} \omega_{\gamma_{y}}^{2}\right]} \cos 3 \omega_{\gamma_{y} t} t \\
& +\frac{3 c e^{2}}{2 b^{3}} \gamma_{\gamma}^{3} \frac{\omega_{\gamma_{y}}^{5}}{\left(\omega_{\gamma_{y}}^{2}-4 \omega_{\beta_{y}}^{2}\right)\left[\left(\omega_{\beta_{y}}^{2}-9 \omega_{\gamma_{y}}^{2}\right)^{2}+9 \frac{c^{2}}{b^{2}} \omega_{\gamma_{y}}^{2}\right]} \sin 3 \omega_{\gamma_{r}} t,
\end{aligned}
$$

where $b=I_{C}, c=\alpha \mathscr{L}_{B}^{2}, e=M_{A} L_{A} L_{\mathrm{cm}}, f=M_{A} L_{\mathrm{cm}}\left(\rho_{C}-\rho_{L}\right)$, and $\omega_{\beta_{y}}^{\prime}=0$ at critical damping.

Sine modulation terms now appear in (53) and (54) because the damping effects introduce phase shifts into the balance beam responses to the pure cosine $\gamma_{x}$ and $\gamma_{y}$ motions. The $\sin \frac{n}{2} \omega_{\gamma} t$ terms are smaller than their equivalent $\cos \frac{n}{2} \omega_{\gamma} \dot{t}$ terms by the ratio $\frac{-2 c}{n b \omega_{\gamma_{r}}}$, where $n=2,4$, or 6 .

The $3 \omega_{\gamma_{v}} t$ modulation terms are negligibly small compared with the $2 \omega_{\gamma_{v}} t$ terms-as was the case for a freely-swinging, undamped balance. The presence of a systematic error term in the equilibrium angle can, in principle, be tested by observing the apparent $\hat{\beta}_{y}$ values for pure $\gamma_{x}$ and $\gamma_{y}$ motions. In practice, however, the over-simplifications of our model may cause problems in interpreting such a test. The modulations of frequency $\frac{\omega_{\gamma_{x}}}{2 \pi}$ can again be minimized by choosing a nominal value of $\hat{\beta}_{y}$ such that terms involving the quantity $M_{A} L_{A} L_{\mathrm{cm}} \hat{\beta}_{y} \gamma_{y}$ are nearly equal in magnitude and opposite in sign to the knife-edges terms which include the factor $M_{A} L_{\mathrm{cm}}\left(\rho_{C}-\rho_{L}\right) \hat{\gamma}_{v^{*}}$

\subsubsection{Experimental Verification}

We have tested some of the predictions of section 5.2 .1 by using a Mettler M5 balance, ${ }^{3}$ which has a velocity-dependent damping produced by an air-dashpot. This balance has a maximum load of $20 \mathrm{~g}$, an onscale range of $20 \mathrm{mg}$, and a readability of $2 \mu \mathrm{g}$. An analysis of some important features of this balance can be found in ref. [7]. The weighing pan is attached to the lower part of the suspension system by a hook and ring, so that the initial motion of the suspension system is that of a double pendulum. This motion quickly dampens to that of a single pendulum-which is a necessary condition for our model. The presence of the hook and ring, however, makes it difficult to generate pure $\gamma_{x}$ or pure $\gamma_{y}$ motion. We therefore temporarily locked the hook and ring together to provide a rigid suspension system for these tests. The tests described below were performed by gently starting the pan swinging at an amplitude of $4 \pm 1 \mathrm{mrad}$ about equilibrium.

What can be inferred from eq (54) about the dynamic behavior of this balance? First, eq (54) can be simplified because $\omega_{\beta_{y}}^{\prime} \approx 0$ and $\omega_{\beta_{\gamma}}^{2}<<\omega_{y}^{2}$ :

$$
\begin{aligned}
\beta_{y}= & \text { const. } \\
& +\frac{e}{b} \hat{\beta}_{y} \gamma_{y}\left[1+\left(\frac{c}{b \omega_{\gamma_{y}}}\right)^{2}\right]^{1 / 2} \cos \left(\omega_{\gamma_{y}} t+\operatorname{Arctan} \frac{c}{b \omega_{\gamma_{v}}}\right) \\
& +\frac{f}{b} \gamma_{y}\left[1+\left(\frac{c}{b \omega_{\gamma_{y}}}\right)^{2}\right]^{1 / 2} \cos \left(\omega_{\gamma_{y} t}+\operatorname{Arctan} \frac{c}{b \omega_{\gamma}}\right) \\
& -\frac{e}{b} \frac{\bar{\gamma}_{y}^{2}}{4}\left[1+\left(\frac{c}{2 b \omega_{\gamma_{y}}}\right)^{2}\right]^{1 / 2} \cos \left(2 \omega_{\gamma_{y}} t+\operatorname{Arctan} \frac{c}{2 b \omega_{\gamma_{y}}}\right) \\
& + \text { higher order terms. }
\end{aligned}
$$

\footnotetext{
* Brand names are ased only for purposes of identification. Such use inplies neither endorsement by the National Bureau of Sundards nor assurance that the equipment is the best arailable.
} 
Term A, the Mathieu term, arises from the tangential acceleration of the swinging pan. Term $B$ is a consequence of having knife edges of finite radius. The last term considered, $\mathrm{C}$, has its origin in the centripetal acceleration of the pan. The latter is the only term of importance in eq (52).

We have made the following estimates for the balance being considered:

$$
\begin{aligned}
e & \sim 5 \times 10^{3} \mathrm{~g} \mathrm{~cm}^{2} \\
|f| & \leqslant 2.5 \times 10^{-2} \mathrm{~g} \mathrm{~cm}^{2} \\
b & \sim 3.5 \times 10^{3} \mathrm{~g} \mathrm{~cm}^{2} \\
c & \sim 5.5 \times 10^{3} \mathrm{~g} \mathrm{~cm}^{2} \mathrm{~s}^{-1} .
\end{aligned}
$$

Also, $\omega_{\gamma_{v}}$ was measured to be $7.8 \mathrm{rad} / \mathrm{s}$.

On the basis of these numbers, one may observe that term $B$ always has much smaller amplitude than term $C$ and is, therefore, always masked. We are left only with terms $A$ and C. Equation (55) predicts that term A will dominate when $\left|\hat{\beta}_{y}\right|>>\bar{\gamma}_{y}$ but that term $C$ will dominate when the inequality is reversed. Thus, under the condition that $\left|\hat{\beta}_{y}\right|>>\bar{\gamma}_{y}$ we would expect an oscillation in the beam at the same frequency as the pan oscillation whereas the oscillation frequency of the beam should double when $\hat{\beta}_{y}=0$. The ratio of the amplitudes in the two regimes is predicted to be

$$
4.4 \hat{\beta} / \tilde{\gamma}_{r}
$$

Note that this result is independent of the estimate of $e$ given above and only weakly dependent on $c$ and $b$. These predictions were tested in the following way.

With the balance arrested, a load of nearly $20 \mathrm{~g}$ was placed on the pan. When the balance was released, the optical scale reading was found to be near zero (i.e., $\hat{\beta}$, was near its maximum value of $+22 \operatorname{mrad}[7]$ ). Pure $\gamma_{x}$ motion produced a small modulation of the balance beam read-out angle, $\beta_{y}$, at a frequency twice that of the suspension system-as predicted by eq (53-at an amplitude of $2 \pm 1 \mu \mathrm{g}$. Pure $\gamma_{y}$ motion yielded a modulation having an amplitude of $32 \pm 2 \mu \mathrm{g}$-with a frequency which was the same as that of the suspension system. The phase shift due to damping effects was too small to observe, so that this modulation was a nearly pure $\cos \omega_{\gamma}, t$ function. Our measurements could have detected a phase shift as small as $\pi / 6$ but eq (55) predicts a phase shift of about $\pi / 16$.

We then changed the sign of $\hat{\beta}_{y}$ by adding $20 \mathrm{mg}$ of tare weights, thereby moving the equilibrium of the beam near to the maximum on-scale reading (i.e., $\hat{\beta}_{y}$ near its minimum value of $-22 \mathrm{mrad}$ [7]). The result of pure $\gamma_{y}$ motion at this position produced a $180^{\circ}$ phase shift in the $\cos \omega_{\gamma} t$ modulation of $\beta_{y}$ as predicted by eq (55).

The magnitude of $M_{A}$ was then reduced by removing $10 \mathrm{mg}$ so that the equilibrium position of the beam was at the middle of the optical scale. At this position, where the beam is nearly horizontal, the Mathieuterm contribution of the $\cos \omega_{\gamma}, t$ modulation nearly vanishes. The $\beta$, modulation now became that of the small $\cos 2 \omega_{\gamma}, t$ contribution (in agreement with the case of $\gamma_{x}$ motion.)

These tests clearly verify several important predictions of eqs (53) and (55). The observed ratio of amplitudes in the two frequency regimes is 16 , which agrees satisfactorily with the value 24 predicted by (56).

\section{Conclusions}

In summation, it will be useful to recall some of the important simplifications in our mathematical model. All knives have been assumed to be symmetrically honed and to roll on perfect flats. The two main knives of the balance are assumed to contact their respective flats.in straight lines which are parallel to each other and to the $x-y$ plane. Many authors have dealt with static errors which result from a violation of those conditions (e.g., $[5,11,12]$ ). An analysis of the corresponding dynamic errors is clearly beyond the scope of this paper. We have also chosen to look at pan oscillations in the context of the small-angle approximation of an idealized balance. While we believe this is appropriate, it should be mentioned that small variations in balance sensitivity 
as a function of $\hat{\beta}_{y}^{2}$ can be observed in many analytical balances having a large on-scale range [7]. Such behavior cannot be predicted from our equations, which ignore angular terms higher than first order.

The above considerations serve as a caveat to the quantitative application of our results to real balances. Nevertheless, the solutions found have proved valuable. The question posed in the introduction has been answered: Two processes are important in analyzing the effect of a swinging pan on balance read-out. One, which has been mentioned in the past [2], is due to centripetal acceleration of the pan. The second-and often predominant-process is a more subtle effect by which the tangential acceleration of the swinging pan leads to a torque on the beam if the latter is not at the gravitational horizon and if the former has a component of motion in the $y-z$ plane. Even a crude approximation of the tangential acceleration (e.g., $a_{T}($ max.) $\sim$ $g \bar{\gamma})$ suggests that its influence will exceed that of the centripetal acceleration if $\hat{\beta}>\bar{\gamma}$. A convincing argument, however, can only be advanced through the rigorous mathematical solution to the equations of motion as presented in section 4.

The disappearance of this second, or "Mathieu" process when the rest-angle of the beam is near gravitational horizon may be used to minimize the effect. Alternatively, it may be possible to use the effect to adjust the beam angle in a class of experiments $[13,14,15]$ where it is advantageous that the beam be parallel to gravitational horizon. Here the radii of the knives must be determined (under load) in a separate experiment [12].

\section{References}

[1] Almer, H.E. One kilogram balance NBS No. 2, J. Res. Nat. Bur. Stand. (U.S.). 76C(1 and 2): 1-10; 1972 January-June. The balance described in this reference is now housed at the Bureau International des Poids et Mesures where it achieves a standard deviation of $1 \mu \mathrm{g}$ in $1 \mathrm{~kg}$.

[2] Bowman, H.A.; Macurdy, L.B. Gimbal device to minimize the effects of off-center loading on balance pans. J. Res. Nat. Bur. Stand. (U.S.). 64C(4): 277-279; 1960 October-December.

[3] Goldstein, H. Classical mechanics. Massachusetts: Addison-Wesley Publishing Co., Inc.; 1950. 399 p. See especially pp 14-18.

[4] Wells, D.A. Theory and problems of Lagrangian dynamics. New York: Schaum Publishing Co.; 1967. See especially pp 58-62.

[5] Bowman, H.A.; Almer, H.E. Minimization of the arrestment error in one-pan, two-knife systems. J. Res. Nat. Bur. Stand. (U.S.). 67C(3): 227-235; 1963 July-September.

[6] Waldersee, J. The effect of the finite radii of the knife edges on the performance of an equi-arm balance. Australian J. Appl. Sc.. 6: $158-166 ; 1955$.

[7] Bowman, Horace A.; Schoonover, Randall M.; [with Appendix by Jones, Mildred W.]. Procedure for high precision density determinations by hydrostatic weighing. J. Res. Nat. Bur. Stand. (U.S.). 71 C(3): 179-198; 1967 July-August.

[8] Stephenson, Reginald J. Mechanics and properties of matter. New York: John Wiley and Sons, Inc.; 1952.371 p. See especially pp 122-129.

[9] Abramowitz, M.; Segun, I.A. Handbook of mathematical functions. New York: Dover Publications, Inc.; $1968.1,046$ p. See especially pp 722-746.

[10] McLachlan, N.W. Theory and application of Mathieu functions. England: Clarendon Press; 1947. 401 p.

[11] Thiesen. J. Etudes sur la balance. Trav. et Mem. Bureau International des Poids et Mesures 5: 3-40; 1886.

[12] Corwin, A.H. Weighing in technique of organic chemistry, part 1 in Physical methods of organic chemistry, vol. 1. Amold Weissberger, ed. New York: Interscience Publishers, Inc.; 1959. 71-129.

[13] Cage, M.E. An analysis of the effects of dynamic and static forces present in the NBS SI volt experiment. Nat. Bur. Stand. (U.S.) NBSIR 80-2143; 1980 September. 218 p.

[14] Olsen, P.T.; Phillips, W.D.; Williams, E.R. A proposed coil system for the improved realization of the absolute ampere. J. Res. Nat. Bur. Stand. (U.S.) 85(4): 257-272; 1980 July-August.

[15] Olsen, P.T.: Cage, M.E.; Phillips, W.D.; Williams, E.R. The realization of the ampere at NBS. IEEE Trans. on Instr. and Meas. 29: $234-237 ; 1980$.

\section{Appendix A. Algebraic substitutions and changes of variables}

This appendix lists all of the algebraic substitutions and changes of variables used in the derivations. Therefore, any equation given in the main text can be readily expressed in terms of the basic quantities $\gamma_{x}$, $\gamma_{y}, \beta, L_{\mathrm{cm}}, L_{A}, \ell_{b}, L_{B}, \mathscr{L}_{B}, \ell_{B}, M_{A}, M_{b}, M_{B}, g, \alpha, t, \rho_{C}, \rho_{L}$, and $\rho_{l}$-as defined in figures $1-4$. 


$$
\begin{aligned}
& \kappa=\left[M_{A} g\left(\rho_{L}+\rho_{C}\right)+M_{b} g\left(\ell_{b}+\rho_{C}\right)+M_{B} g\left(\ell_{B}+\rho_{C}\right)\right] \\
& S=\frac{1}{\kappa} \\
& F_{\alpha}=-\alpha \mathscr{L}_{B} \dot{\beta}_{y} \\
& I_{C}=M_{\mathrm{A}} L_{A}^{2}+\left[\left(I_{b}\right)_{\mathrm{cm}}+M_{b} \ell_{\mathrm{cm}}^{2}\right]+\left[\left(I_{B}\right)_{\mathrm{cm}}+M_{B}\left(L_{B}^{2}+\ell_{B}^{2}\right)\right] \\
& I_{P}=\left(I_{S}\right)_{c m}+M_{A} L_{c m}^{2} \\
& I_{P}^{\prime}=\left(I_{\mathrm{s}}^{\prime}\right)_{\mathrm{cm}}+M_{A} L_{\mathrm{cm}}^{2} \\
& \omega_{\gamma_{x}}^{2}=\frac{\left[M_{A} g L_{c m}\right]}{I_{P}^{\prime}} \\
& \omega_{\gamma_{r}}^{2}=\frac{\left[M_{A} g L_{c m}\right]}{I_{P}} \\
& \omega_{\gamma_{x}}=\frac{2 \pi}{P_{\gamma_{x}}} \\
& \omega_{\gamma_{v}}=\frac{2 \pi}{P_{\gamma_{v}}} \\
& \gamma_{x}=-\bar{\gamma}_{x} \cos \omega_{\gamma_{x}} t \\
& \gamma_{y}=-\gamma_{y} \cos \omega_{\gamma_{y}} \\
& b=I_{C} \\
& c=\alpha \mathscr{S}_{B}^{2} \\
& d=\left[M_{B} g L_{B}-M_{A} g L_{A}\right] \\
& e=M_{A} L_{A} L_{\mathrm{cm}} \\
& f=M_{A} L_{\mathrm{cm}}\left(\rho_{C}-\rho_{L}\right) \\
& \hat{\beta}_{y}=\frac{d}{\kappa}=\frac{\left[M_{B} g L_{B}-M_{A} g L_{A}\right]}{\left[M_{A} g\left(\rho_{L}+\rho_{C}\right)+M_{b} g\left(\ell_{b}+\rho_{C}\right)+M_{B} g\left(\ell_{B}+\rho_{C}\right)\right]} \\
& \omega_{\beta_{\gamma}}^{\prime}=\sqrt{\frac{\kappa}{b}-\frac{c^{2}}{4 b^{2}}}=\frac{2 \pi}{P_{\beta}^{\prime}} \\
& \omega_{\beta_{v}}=\sqrt{\frac{k}{b}}=\frac{2 \pi}{P_{B}} \\
& \bar{u}=\frac{c}{b}=\frac{\alpha \mathscr{L}_{B}^{2}}{I_{C}} \\
& v=\sqrt{\frac{\kappa}{b}}=\omega_{\beta} . \\
& w=\frac{e}{b} \gamma_{x}^{2} \omega_{\gamma_{x}}^{2}=\frac{M_{A} L_{A} L_{\mathrm{cm}}}{l_{C}} \gamma_{x}^{2} \omega_{\gamma_{x}}^{2} \\
& k=2 \omega_{\gamma_{s}} \\
& D=\frac{\bar{u} w k}{\left[\left(v^{2}-k^{2}\right)^{2}+\bar{u}^{2} k^{2}\right]} \\
& E=\frac{u\left(v^{2}-k^{2}\right)}{\left[\left(v^{2}-k^{2}\right)^{2}+\bar{u}^{2} k^{2}\right]}
\end{aligned}
$$




$$
\begin{aligned}
& z=\frac{\omega_{y_{x}}}{2} t \\
& \bar{k}=\frac{c}{b \omega_{\gamma_{y}}}=\frac{\alpha \mathscr{L}_{B}^{2}}{l_{C} \omega_{\gamma_{y}}} \\
& \bar{a}=\frac{4 \omega_{\beta_{\gamma}}^{2}}{\omega_{\gamma_{y}}^{2}} \\
& q=-2 \frac{e}{b} \tilde{\gamma}_{y}=-2 \frac{M_{A} L_{A} L_{\mathrm{cm}}}{l_{C}} \bar{\gamma}_{y} \\
& h=\frac{4 d}{\omega_{\gamma_{y}}^{2} b}=4 \frac{\left[M_{B} g L_{B}-M_{A} g L_{A}\right]}{I_{C} \omega_{\gamma_{v}}^{2}} \\
& \ell=4 \frac{e}{b} \bar{\gamma}_{y}^{2}=4 \frac{M_{A} L_{A} L_{\mathrm{cm}}}{I_{C}} \hat{\gamma}_{y}^{2} \\
& m=4 \frac{f}{b} \bar{\gamma}_{y}=4 \frac{M_{A} L_{\mathrm{cm}}}{I_{C}}\left(\rho_{C}-\rho_{L}\right) \bar{\gamma}_{y} \\
& \bar{f}(z)=h+\ell \cos 4 z-m \cos 2 z \\
& \beta_{y}=e^{-i z} u(z) \\
& a=\bar{a}-\bar{k}^{2}=\frac{4}{\omega_{\gamma_{,}}^{2}}\left[\frac{\kappa}{b}-\frac{c^{2}}{4 b^{2}}\right]=4 \frac{\omega_{\beta_{\gamma}}^{\prime 2}}{\omega_{\gamma_{1}}^{2}} \\
& \omega_{\beta_{y}}^{\prime}=0 \quad(\text { if } a=0) \\
& \bar{q}=\frac{q}{2\left(1-\nu^{2}\right)} \approx-\frac{e}{b} \frac{\omega_{\gamma_{v}}^{2} \bar{\gamma}_{\nu}}{\left(\omega_{\gamma_{v}}^{2}-4 \omega_{\beta_{s}}^{\prime 2}\right)} \\
& \nu \approx \sqrt{a} \approx 2 \frac{\omega_{\beta_{y}}^{\prime}}{\omega_{\gamma_{1}}} \quad(\text { if } a>0) \\
& v \approx \frac{q}{\sqrt{2}} \approx \sqrt{2} \bar{q} \approx-\sqrt{2} \frac{e}{b} \bar{\gamma}_{y} \quad \text { (if } a=0 \text { ) } \\
& u_{1}=\cos v z-\bar{q} \cos v z \cos 2 z-v \bar{q} \sin v z \sin 2 z \\
& u_{2}=\sin v z-\bar{q} \sin v z \cos 2 z+v \bar{q} \cos v z \sin 2 z \\
& \bar{c}^{2}=u_{1} u_{2}^{\prime}-u_{2} u_{1}^{\prime}=v \\
& v z=\omega_{\beta_{r}}^{\prime} t \\
& 2 z=\omega_{\gamma_{x}} t \\
& \overrightarrow{k z}=\frac{c t}{2 b} \\
& v \bar{q}=-2 \frac{e}{b} \frac{\omega_{\gamma_{r}} \omega_{\beta_{r}}^{\prime} \bar{\gamma}_{\gamma}}{\left(\omega_{\gamma_{v}}^{2}-4 \omega_{\beta_{1}}^{\prime 2}\right)}
\end{aligned}
$$

\section{Appendix B}

An aid to identifying terms in eq (50)

This appendix provides some relationships that can aid the reader in transforming the terms in eq. (50) for $\gamma_{y}$ and $\beta_{y}$ motions into the notation used in (28) for $\gamma_{x}$ and $\beta_{y}$ motions. The combination

$$
\frac{X}{\left[k^{2}+(v+n)^{2}\right]}+\frac{Y}{\left[k^{2}+(v-n)^{2}\right]}
$$


often occurs in (50), where $n=0,2,4$ or 6 . By using the common denominator

$$
\left[\left(\bar{a}-n^{2}\right)^{2}-4 n^{2} a\right]=\frac{16}{\omega_{\gamma,}^{4}}\left[\left(\omega_{\beta, y}^{2}-\frac{n^{2}}{4} \omega_{\gamma_{y}}^{2}\right)^{2}+\frac{n^{2}}{4} \frac{c^{2}}{b^{2}} \omega_{\gamma_{y}}^{2}\right]
$$

(Bl) can be written as

$$
-\frac{n v \bar{k} \omega_{\gamma_{y}}^{4}}{4\left[\left(\omega_{\beta_{y}}^{2}-\frac{n^{2}}{4} \omega_{\gamma_{y}}^{2}\right)^{2}+\frac{n^{2} c^{2}}{4 b^{2}} \omega_{\gamma_{y}}^{2}\right]} \quad \text { (if } X=\bar{k} \text { and } Y=-\bar{k} \text { ); }
$$

as

$$
\left.\frac{\bar{k}\left(4 \omega_{\beta_{\gamma}}^{2}+n^{2} \omega_{\gamma_{\gamma}}^{2}\right) \omega_{\gamma_{v}}^{2}}{8\left[\left(\omega_{\beta_{\gamma}}^{2}-\frac{n^{2}}{4} \omega_{\gamma_{\gamma}}^{2}\right)^{2}+\frac{n^{2}}{4} \frac{c^{2}}{b^{2}} \omega_{\gamma_{\gamma}}^{2}\right]} \quad \text { (if } X=\bar{k} \text { and } Y=\bar{k}\right)
$$

as

$$
\left.\frac{v\left(4 \omega_{\beta_{\gamma}}^{2}-n^{2} \omega_{\gamma_{\gamma}}^{2}\right) \omega_{\gamma_{\gamma}}^{2}}{8\left[\left(\omega_{\beta_{\gamma}}^{2}-\frac{n^{2}}{4} \omega_{\gamma_{\gamma}}^{2}\right)^{2}+\frac{n^{2}}{4} \frac{c^{2}}{b^{2}} \omega_{\gamma_{\gamma}}^{2}\right]} \quad \text { if } X=(\nu+n) \text { and } Y=(\nu-n)\right)
$$

or as

$$
\left.\frac{n}{8} \frac{\left(4 \omega_{\beta_{\gamma}}^{2}+n^{2} \omega_{\gamma_{\gamma}}^{2}-8 \omega_{\beta_{\gamma}}^{2}\right) \omega_{\gamma_{\gamma}}^{2}}{\left[\left(\omega_{\beta_{\gamma}}^{2}-\frac{n^{2}}{4} \omega_{\gamma_{y}}^{2}\right)^{2}+\frac{n^{2}}{4} \frac{c^{2}}{b^{2}} \omega_{\gamma_{y}}^{2}\right]} \quad \text { (if } X=(\nu+n) \text { and } Y=-(\nu-n)\right) \text {. }
$$

With the help of eqs (B1)-(B5) and Appendix A, the terms of (50) can be expressed in the notation of (28). For example:

$$
\frac{m \bar{k}}{2 \nu} \frac{\sin 2 z}{\left[\bar{k}^{2}+(\nu+2)^{2}\right]}-\frac{m \bar{k} \sin 2 z}{2 \nu\left[\bar{k}^{2}+(\nu-2)^{2}\right]}=-\frac{c f}{b^{2}} \gamma_{y} \frac{\omega_{\gamma_{y}}^{3}}{\left[\left(\omega_{\beta_{y}}^{2}-\omega_{\gamma_{j}}^{2}\right)^{2}+\frac{c^{2}}{b^{2}} \omega_{\gamma_{y}}^{2}\right]} \sin \omega_{\gamma_{y}} t,
$$

and

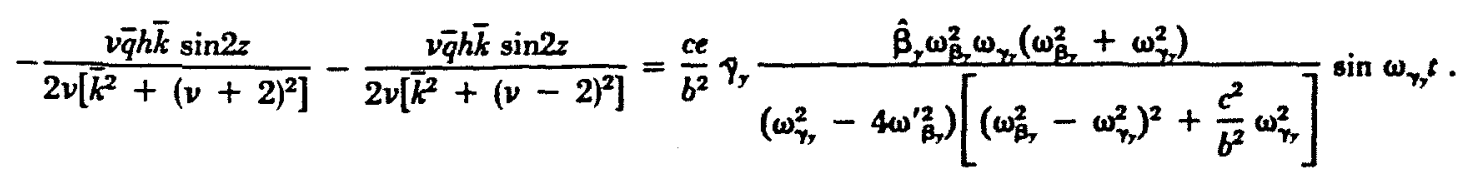

